\title{
Notch 1 Receptor, Delta 1 Ligand and HES 1 Transcription Factor are Expressed in the Lining Epithelium of Periapical Cysts (Preliminary Study)
}

\author{
Meliou $\mathrm{E}^{*}, 1$, Kerezoudis $\mathrm{NP}^{1}$, Tosios $\mathrm{KI}^{2}$ and Kiaris $\mathrm{H}^{3}$ \\ ${ }^{1}$ Dept. of Endodontology, Dental School, University of Athens, Greece, ${ }^{2}$ Dept. of Oral Pathology and Surgery, Dental \\ School, University of Athens, Greece, ${ }^{3}$ Dept. of Biochemistry, Medical School, University of Athens, Greece
}

\begin{abstract}
Periapical cyst is a chronic inflammatory disorder of periradicular tissues. The precise pathological mechanisms involved in periapical cyst enlargement remain unclear. Notch signaling is an evolutionarily conserved pathway with a regulatory role in cell fate decisions during development and in carcinogenesis. To date, there are no published data available on the expression of Notch signaling components in periapical cysts or any other jaw cyst. In this immunohistochemical study we have examined the expression of the receptor Notch 1, the ligand Delta 1 and the transcription factor HES 1 in the epithelium of well defined periapical cysts. Immunostaining reaction of Notch 1, Delta 1 and HES 1 was observed in the cytoplasm and/or the cytoplasmic membrane and occasionally in the nucleus in the majority of epithelial cells of all periapical cysts. The present observations indicate that Notch pathway is active in the epithelium of periapical cysts. It can be speculated that activation of epithelial cells of periapical cysts is associated with activation of Notch pathway and imply involvement of this pathway in periapical cyst growth and expansion.
\end{abstract}

Keywords: Notch 1, Delta 1, HES 1, epithelium, periapical cyst.

\section{INTRODUCTION}

Apical periodontitis is a chronic inflammatory disorder of periradicular tissues caused by persistent microbial infection within the root canal system of the affected tooth. The reported incidence of cysts among apical periodontitis lesions varies from $6 \%$ to $55 \%$, but standardized histopathological studies have shown that only $15 \%-17 \%$ of those lesions were actually periapical cysts [1]. The mechanisms underlying the development and expansion of periapical cysts have not been fully elucidated. Several theories have been proposed to explain the formation of a periapical cyst, i.e. the "nutritional deficiency theory", the "abscess theory" and the newly proposed "merging of epithelial strands" theory [2]. Generally, it is thought that the epithelial cell rests of Malassez proliferate under the influence of mediators and growth factors released by various cells residing in the periapical tissues, forming a stratified squamous epithelium-lined cavity. Rarely, however, periapical cysts have also been found to be lined partially or predominantly by ciliated columnar cells that may have respiratory origin [3]. Regarding cyst expansion, the osmotic pressure exerted on the cystic lumen has been implicated as a main reason in the past, but nowadays research has been focused on molecular mechanisms of epithelial cell proliferation since, certain growth factors and cytokines are expressed in the cyst lining epithelium $[1,2]$.

Notch is one of the fundamental signaling systems that regulate embryonic development and adult tissue development. Notch was first identified in 1919 as a mutant gene that causes "notches" at the wing margin of the Drosophila

*Address correspondence to this author at the 39 P. Ioakeim Street, 10675Athens, Greece; Tel: -0030-2107297612;

E-mail: emeliou@dent.uoa.gr fly [4]. In mammals, four Notch receptors (Notch 1- Notch 4) and five structurally similar Notch ligands (Delta 1, Delta 3, Delta 4, Jagged 1 and Jagged 2) have been identified. Notch receptors share a high degree of structural homology between members as well as across species. It is a singlepass transmembrane protein consisting of a large extracellular domain with 36 EGF repeats, involved in ligand binding and a cytoplasmic domain involved in signal transduction. The Notch ligands are also single-pass membrane-bound proteins. They also have a variable number of EGF-like repeats for binding with the receptor. EGF repeats are found in many growth factor and receptor molecules and participate in protein association [4]. The most well-defined targets of the Notch pathway are the HES (Hairy and Enhancer of Split) family of basic helix-loop-helix transcription factors [5].

Notch signaling has been identified in vertebrate central nervous system development, somitogenesis, cardiovascular and endocrine development. Notch also has a role in tumorogenesis where it can act as an oncogene or as a tumor suppressor, depending on strength, timing, cell type and context of the signal [6]. In the field of dentistry, Notch signaling is expressed in epithelial-mesenchymal interactions during odontogenesis [7], in the proliferation and differentiation process of cultured dental pulp stem cells into odontoblast-like cells in differentiation-inductive environments [8] and are associated with dental pulp pathology after injury, carious lesions [9] or pulp capping [10]. In tooth germs, Notch receptors are expressed in the odontogenic epithelium (except for the inner enamel epithelium), and Notch ligands are expressed in lower concentrations in the inner enamel epithelium than in other epithelial components [11]. They, also, regulate oral epithelia during palate development to prevent premature palatal shelf-adhesion to other 
oral tissues and to facilitate normal adhesion between the elevated palatal shelves [12]. When compared to normal tissues, Notch signaling molecules demonstrate an increased expression in squamous cell carcinomas of the head and neck (HNSCC) [13] and in ameloblastomas [11, 14].

Since Notch signaling participates in the differentiation and proliferation of epithelial cells, in normal and neoplastic tissues, we investigated the expression of the receptor Notch 1 , the ligand Delta 1 and the transcription factor HES 1 in the epithelial cells of periapical cysts. To our knowledge, there is no published study on Notch signaling expression in periapical cysts or any other jaw cyst.

\section{MATERIAL AND METHODOLOGY}

\section{Study Group}

Thirty periapical cysts from 30 patients archived in the Department of Oral Pathology and Surgery during the randomly selected period 1990 to 1995 , were used in this study. The study was approved by the official ethical committee of the Dental School (94-30/09/08). The patients' age was 1773 years (median 38 years, interquarteal range-IQR 32-50 years). Ten patients $(33.3 \%)$ were female and twenty were $(66.7 \%)$ male. There was no statistical difference in age between sexes $(\mathrm{p}=0.182)$. All lesions were fixed in $10 \%$ neutral buffered formalin solution (24-48h) and embedded in paraffin (FFPE). Specimens with a diagnosis of periapical cyst were included. Diagnosis was established on conventional hematoxylin and eosin stained tissue sections. All the selected lesions showed a well-defined cystic sac lined by nonkeratinized squamous epithelium, with slight inflammation (category 1 by Orstavik and Mjor [15]) in the underlying connective tissue. Specimens with intense inflammatory infiltration were not included.

\section{Immunohistochemistry}

Immunohistochemistry was performed on consecutive FFPE tissue sections. The following primary antibodies were utilized: rat anti-human Notch 1 monoclonal antibody (bTAN 20, 1:10, Developmental Studies Hybridoma Bank, University of Iowa, Iowa, USA), rabbit anti-Mouse Delta 1 antibody (catalog No. 600-401-858, 1:50, Rockland Immunochemicals, USA) and anti-rat HES 1 monoclononal antibody (NM1, 1:100, MBL $^{\mathrm{TM}}$ Int. Inc., Woburn, MA). A standard avidin-biotine-peroxidase staining method was applied. In summary, 3-4 $\mu \mathrm{m}$ tissue sections were cut and dried overnight at $56-58^{\circ} \mathrm{C}$. After deparaffinization, endogenous peroxidase was blocked with $37 \% \mathrm{H}_{2} \mathrm{O}_{2}(10 \mathrm{ml}$ in $90 \mathrm{ml}$ demineralised water, 30 minutes in dark place) and antigen retrieval was achieved with $10 \%$ Trilogy $^{\mathrm{TM}}$ (CMX833-C, Cell Marque Corporation, Roklin, CA) in demineralised water in a conventional steamer for 2 rounds of 40 minutes each. After application of the primary antibodies overnight at $4^{\circ} \mathrm{C}$, immunostaining was developed with Dako Real ${ }^{\mathrm{TM}}$ EnVi$\operatorname{sion}^{\mathrm{TM}}$ System (5007, Dako, Glostrup, DE) with 3,3'diaminobenzidine tetrahydrochloride (DAB, Sigma-Aldrich Corp. St. Louis, MO, USA) for Notch 1, that gives a brown colour or EnVision ${ }^{\mathrm{TM}}$ G|2 System/AP (K5355, Dako, Glostrup, DE) with alkaline phosphatase (Sigma-Aldrich Corp. St. Louis, MO, USA) for Delta 1 and HES 1, that gives intense red colour. Sections were finally counterstained with Mayer's Hematoxylin (AR106-Artisan ${ }^{\mathrm{TM}}$, Dako North
America, Inc., California, USA) for 45 seconds, cleared and mounted. Breast and lung [6] tumors served as positive controls. Substitution of the primary antibody with non-immune serum of the same species served as a negative control.

\section{Computerized Image Analysis (CIA) Method}

Immunohistochemical expression pattern of Notch 1, Delta 1 and HES 1 was analyzed after observation using an optical microscope. Quantitative evaluation of staining intensity levels of the corresponding markers was performed by a computerized semi automated image analysis system (Hardware: Mobile Intel Pentium Dual core/ Digital Still Camera SONY CyberShot DSC-P73 (1024X768), microscope Olympus CX-31, Software: Windows XP/Image Pro Plus version 5.1-Media Cybernetics 2000) (Fig. 1) [16]. A macro was implemented based on the control group (breast and lung cancerous epithelia) and protein expression of the lesions (cyst specimens). Staining intensity values were ranged between 0 (black-highest expression level) and 255

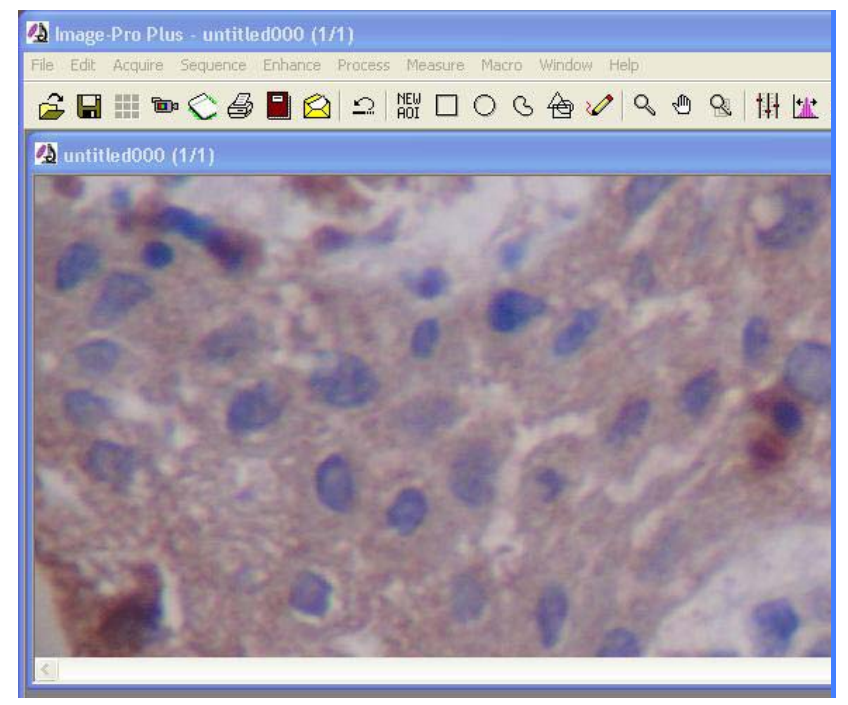

(a)

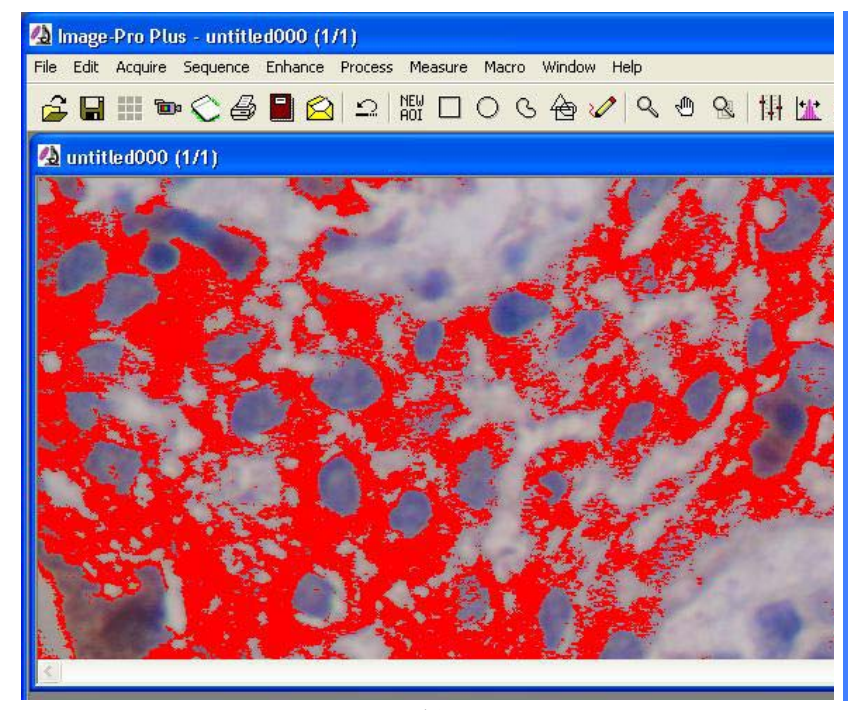

(b)

Fig. (1). Computerized image analysis process. Red signals (b) represent the stained cytoplasm/membrane (a). 
Table 1. Mean Values of Notch 1, Delta 1 and HES 1 Obtained from Computerized Image Analysis System ( $S=$ strong, $\mathrm{M}=$ moderate and $\mathrm{W}=$ weak staining)

\begin{tabular}{|c|c|c|c|}
\hline Case. no & Notch 1 & Delta 1 & HES 1 \\
\hline 1 & M (126) & $\mathrm{W}(178,75)$ & M (141.25) \\
\hline 2 & M (129) & M (141) & M (120.5) \\
\hline 3 & W (154.25) & $\mathrm{M}(128.25)$ & M (120.25) \\
\hline 4 & S (118) & W (167.5) & M (139.75) \\
\hline 5 & S (102) & $\mathrm{M}(143.75)$ & M (149.5) \\
\hline 6 & M (133) & M (142.5) & M (127.25) \\
\hline 7 & $S(113.75)$ & $\mathrm{W}(152.25)$ & M (135) \\
\hline 8 & M (144.5) & $\mathrm{M}(142.75)$ & M (143.5) \\
\hline 9 & M (141.75) & W (167.25) & M (139.5) \\
\hline 10 & S (107.75) & $\mathrm{M}(144.75)$ & M (142.25) \\
\hline 11 & $\mathrm{~S}(92.25)$ & M (144) & S (118) \\
\hline 12 & S (118.5) & W (159.5) & W (161) \\
\hline 13 & W (169) & $\mathrm{W}(171.25)$ & W (164) \\
\hline 14 & M (129.25) & M (143.25) & M (145.75) \\
\hline 15 & M (124.5) & M (148) & W (167.5) \\
\hline 16 & $S(116.25)$ & M (141) & W (155.5) \\
\hline 17 & M (127.75) & M (146.5) & M (137.75) \\
\hline 18 & S (110) & W (157) & M (141.25) \\
\hline 19 & S (96) & M (138.25) & $S(115)$ \\
\hline 20 & M (138) & W (156) & M (135.25) \\
\hline 21 & M (138.5) & M (128.75) & M (141) \\
\hline 22 & S (103.75) & M (125.75) & S (111.25) \\
\hline 23 & W (172) & M (131.25) & S (119.75) \\
\hline 24 & S (103.75) & W (166) & M (121.75) \\
\hline 25 & S (117.75) & W (152) & W (166.25) \\
\hline 26 & S (115) & M (139.25) & M (134.75) \\
\hline 27 & M (125.5) & W (154.5) & W (165.5) \\
\hline 28 & S (106.25) & W (161.75) & W (153.25) \\
\hline 29 & M (126.75) & M (141.5) & M (137) \\
\hline 30 & S (103.75) & W (152.5) & M (136.75) \\
\hline
\end{tabular}

0-120 strong staining; $121-150$ moderate staining; $151-255$ weak staining [17].

(white-complete absence of protein expression) demonstrating strong expression (values decreasing to 0) and loss of expression (values increasing to 255). Interpretation of staining intensity values (range 0-255) was made according to Tsiambas et al. methodology [17]. All of the numerical results were filed in Microsoft Excel sheets and demonstrated in Table $\mathbf{1}$.

\section{Statistical Analysis}

Mean values obtained by computerized image analysis method were subjected to statistical analysis by the applica- tion of frequencies and percentages. Differences in distribution depending on age or sex were evaluated by nonparametric Mann Whitney U-test and Kruskal Wallis test. The regularity of distributions was examined graphically (histograms) and by the Shapiro-Wilk test.

\section{RESULTS}

Immunostaining for Notch 1, Delta 1 and HES 1 was seen in the cytoplasm of cancer cells of breast and lung tumors utilized as controls, but not in the stromal cells (Fig. 2a,d,g). Immunoreactivity for Notch 1 (Fig. 2b,c), Delta 1 (Fig. 2e,f) and HES 1 (Fig. 2g,i) was seen in all layers of the lining epithelium of the periapical cysts examined, but not in the underlying connective tissue, and was mostly cytoplasmic. Cell membrane staining was seen in 1 case for Notch 1 , 3 cases for Delta 1, and 12 cases for HES 1 . Nuclear staining was observed occasionally in all cases. Immunostaining of all antibodies was also noticed in the inflammatory cells infiltrating the underlying connective tissue in periapical cysts.

Notch 1 showed strong expression in 15/30 cases (50\%), moderate expression in $22 / 30$ cases $(40 \%)$ and weak expression in $3 / 30$ cases $(10 \%)$. Delta 1 immunostaining was only moderate in $17 / 30$ cases $(56.67 \%)$ and weak in $13 / 30$ cases $(43.33 \%)$. The HES 1 expression appeared to be strong in $4 / 30$ cases $(13.33 \%)$, moderate in $19 / 30$ cases $(63.33 \%)$ and weak in $7 / 30$ cases $(23.33 \%)$.

Statistical analysis revealed that there was no statistical correlation between the staining intensity of the antibodies and the sex or the age of the study group. There was no statistical difference in age between men and women $(\mathrm{p}=0.222)$.

\section{DISCUSSION}

In the present study, immunohistochemistry was performed in order to detect the localization of Notch signaling proteins in the epithelial cells and their distribution in the cytoplasm, the cell membrane or the nuclei of these cells. Staining was evaluated by computerized image analysis that offered accurate and fast means of estimation, depending on some objective parameters, such as optical density [16, 17]. Thus, this method was better than conventional eye microscopy in handling and evaluating a large number of immunohistochemical data $[16,17]$.

In particular, the expression of the Notch 1 receptor, Delta 1 ligand and HES 1 downstream target in epithelium of non-inflamed human periapical cysts was examined. Even though Notch 1 is required for conventional activation of the Notch 1 signaling pathway, its expression does not necessarily imply that the pathway has been activated downstream [18]. Therefore, in order to determine Notch 1 pathway activation, the HES-1 expression was also examined. This molecule is the best characterized member of the mammalian HES family and has been shown to be the most important downstream target of Notch signaling. The results of the present study show HES-1 expression in the lining epithelium of periapical cysts, suggesting a possible activation of Notch signaling pathway. The expression of other Notch signaling components, such as other receptors, ligands or downstream target genes, needs further study. 


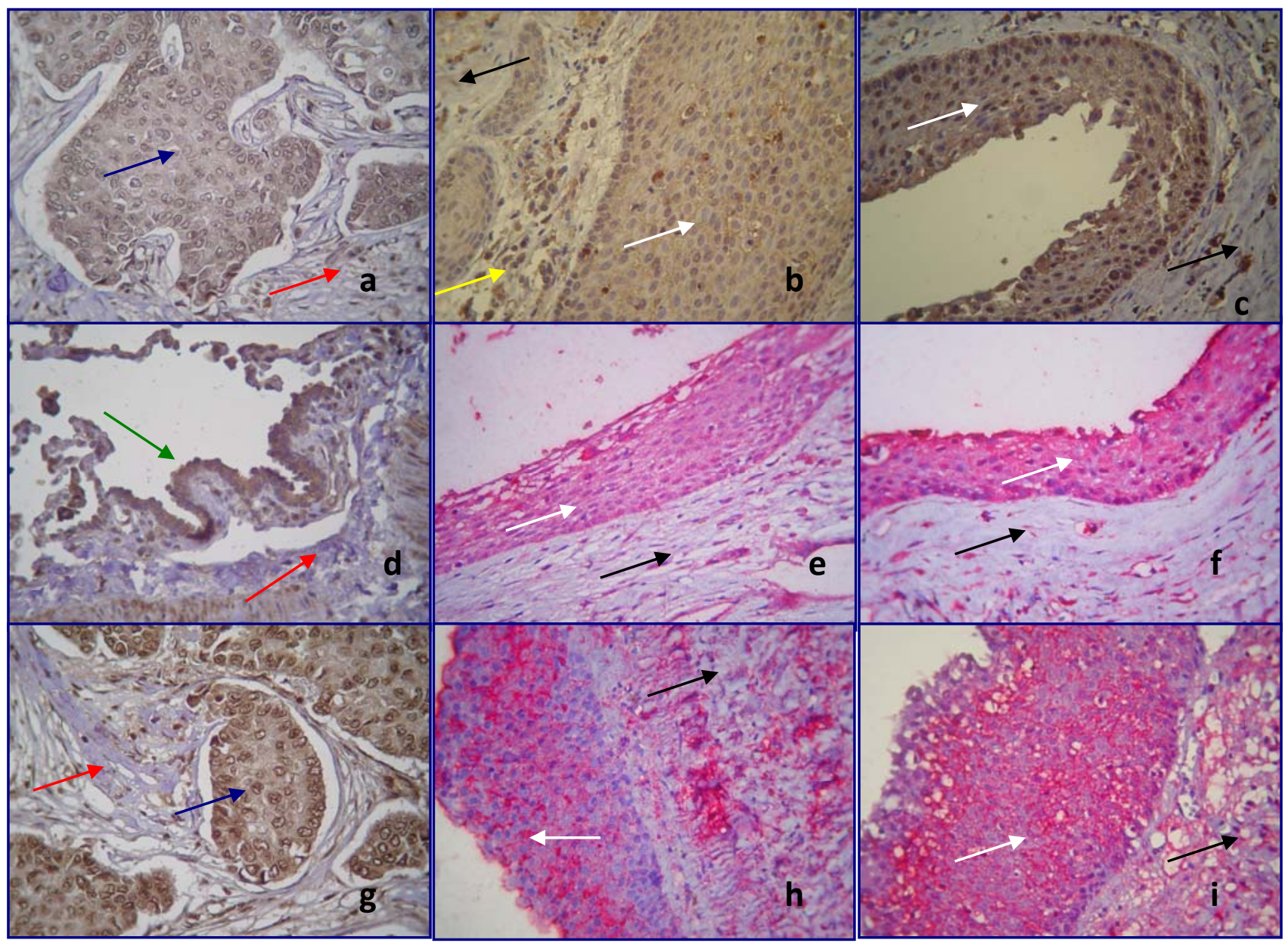

Fig. (2). Immunohistochemical localization of Notch 1 (a,b,c), Delta $1(\mathrm{~d}, \mathrm{e}, \mathrm{f})$ and HES 1 (g,h,i) in positive controls (a,d,g) and in epithelial cells of human periapical cysts (b,c,e,f,h,i) (magnification x400). Staining is shown in brown (chromogen DAB) and red (chromogen alkaline phosphatase). White arrows show the lining epithelium of periapical cysts, while black arrows show the underlining connective tissue. Blue arrows demonstrate the human breast tumor cells, green arrow the human lung tumor cells, served as positive control, while red arrows reveal the stromal cells of the connective tissues.

Notch 1 positive staining (a) is distributed in the human breast tumor cells. No immunoreactivity was observed in the stromal cells. Notch 1 moderate (b) and strong (c) staining is observed in the cytoplasm of epithelial cells of periapical cyst. The underlying connective tissue remains negative for Notch 1 . Notice that the inflammatory cells infiltrated in the connective tissue are Notch 1 positive (yellow arrow).

Positive immunoreacivity for Delta 1 is shown in the human lung tumor cells (d). Weak (e) and moderate (f) cytoplasmic Delta 1 staining is detected in periapical cyst epithelium. HES 1 cytoplasmic staining is noticed in human breast tumor cells (g). HES 1 staining is localized in the cell membrane of epithelial cells $(\mathbf{h}, \mathbf{i})$.

All proteins studied were found in the lining epithelium of the cysts examined, although their topographical distribution and their staining intensity showed variations. In particular, Notch 1, Delta 1 and HES 1 showed mainly cytoplasmic immunoreactivity, membranous positivity was more common for HES 1, while nuclear staining was identified occasionally in all cases. Distribution of Notch signaling components in different cell elements has been described in other studies, i.e. Jagged-2 has been found in the cell membrane, peripheral cytoplasm and entire cytoplasm in multiple myeloma cells [19], and Delta 4 in the cell membrane and the cytoplasm of the luminal and glandular epithelium of the endometrium [20]. Although Notch ligands are anchored on the cell membrane restricting ligand/receptor association only between neighbouring cells, it has also been suggested that receptors and ligands may interact in the same cell forming homomeric or heteromeric complexes [21]. The ho- momeric complexes are present in cell membrane, while the heteromeric complexes probably remain within the cell and do not appear on the cell surface, so a significant portion of ligand and receptor is retained in the cytoplasm.

In the presents study, Notch 1 showed intense expression in most cases. Delta 1 expression was moderate to weak, while HES 1 expression appeared to be mainly moderate. It is suggested that Notch 1 can receive activating signals from other ligands, such as Delta 3, 4 or Jagged 1,2, which were not investigated in this study. Delta 1 and Jagged 1, 2 ligands are known to bind at least Notch 1 and Notch 2 receptors [22], while both Delta 1 and Jagged 1 can activate Notch 1 efficiently [23]. Thus, in non-inflamed human periapical cysts there may be a convergence of multiple signals to Notch 1 that may in turn amplify its expression. Another explanation for the difference in staining intensities may be attributed to the antibody intrinsic binding abilities and not 
to absolute number of ligands and receptors per se. Based on that, a descriptive statistical analysis between antibodies was performed and no effort was made to correlate the differences in staining expression levels between them.

Notch signaling molecules were absent from fibroblasts in breast and lung tumors that served as positive controls, as well as in the underlying connective tissue in periapical cysts. This finding is in agreement with previous findings showing absence of Notch 2 reactivity in pulp fibroblasts of embryonic or adult human teeth (intact, injured or carious) [9].

In contrast, the inflammatory cells infiltrating the connective tissue were positive for Notch signaling. Such a positive staining for inflammatory cells in the pulp of carious permanent human teeth has been reported previously [9]. It is well established that Notch signaling plays a critical role in differentiation, proliferation and apoptosis of T cells [24]. Based on this knowledge and to avoid misinterpretation of the results, only periapical cysts with none or slight inflammatory infiltration in the underlying connective tissue were studied.

Localization of Notch 1 and Delta 1 in the same cell has been described in cancer cells of the prostate as well as metastatic cells, suggesting that Notch signaling can be successfully activated without the need to co-culture them with Notch ligand-expressing cells [25]. This is in contrast with the expression pattern of Notch and Delta during tissue development, where they are mainly complementary and confined to different cell layers, implying Notch pathway involvement in cell-cell interactions that lead to cytodifferentiation and tissue development. For example during odontogenesis, Delta 1 is expressed in differentiating odontoblasts and Notch 1 in the adjacent cells of dental papilla that will form the subodontoblastic layer [7]. In our study, Notch signaling components were localized in the same tissue, the lining epithelium of periapical cysts, but not in the adjacent connective tissue. This finding may be an indication of "independency" of these cells in their proliferation.

Considering the established role of Notch signaling in carcinogenesis, the activation of Notch pathway in the epithelial cells of periapical cysts is consistent with the notion that these cells are in a dynamic state. Our findings, as well as the reported expression of other endogenous regulatory mediators such as cytokines, prostaglandins, interleukins (IL-1, IL-6), the keratinocyte growth factor (KGF) and the epidermal growth factor (EGF) receptor [2] in periapical cystic wall, indicate that the growth and expansion of cysts may be related to epithelial cell proliferation caused by those factors. Tummers and Thesleff [26] reported the expression of Notch 2 and Lfng in Hertwig's epithelial root sheath in vole molars. Since it is believed that the lining epithelium of periapical cysts derives from the epithelial cell rests of Malassez, Notch signaling may be a possible molecular pathway for the generation of periapical cysts.

It should be noticed that Notch signaling molecules have been implicated in the pathogenesis of autosomal dominant polycystic kidney disease (ADPKD) [27]. In ADPKD abnormalities the proliferation and apoptosis of the cells of tubular epithelium result in the formation of cysts. Although, to the best of our knowledge, the pathogenesis of odontogenic cysts has not been associated with that of ADPKD, this is issue merits further research.

\section{CONCLUSIONS}

With the limitations of this study, it was found that the cells of the lining epithelium of the periapical cysts studied expressed the Notch signaling components Notch 1 receptor, Delta 1 ligand, and HES 1 transcription factor, implying the activation of Notch pathway in the cystic epithelium and its involvement in epithelial cells' proliferation.

\section{ACKNOWLEDGEMENTS}

The rat anti-human Notch 1 monoclonal antibody (catalog No: bTAN 20) was obtained from the Developmental Studies Hybridoma Bank developed under the auspices of the NICHD and maintained by The University of Iowa, Department of Biological Sciences, Iowa City, IA 52242.

This study was financially supported by a grant from the University of Athens (70/4/6450).

The authors acknowledge the expertise skill of Captain (MC) E. Tsiambas, MD, Ph.D., Department of Pathology, Tissue Microarrays and Computerized Image Analysis Laboratory, 417 VA Hospital.

\section{REFERENCES}

[1] Nair PNR, Pajarola G, Schroeder HE. Types and incidence of human periapical lesions obtained with extracted teeth. Oral Surg Oral Med Oral Pathol 1996; 81: 93-102.

[2] Lin LM, Huang GT, Rosenberg PA. Proliferation of epithelial cell rests, formation of apical cysts, and regression of apical cysts after periapical wound healing. J Endod 2007; 33: 908-16.

[3] Nair PNR, Pajarola G, Luder HU. Ciliated epithelium-lined radicular cysts. Oral Surg Oral Med Oral Pathol Oral Radiol Endod 2002; 94: 485-93.

[4] Artavanis-Tsakonas S, Rand MD, Lake RJ. Notch signaling: cell fate control and signal interaction in development. Science 1999; 284: 770-6.

[5] Iso T, Kedes L, Hamamori Y. HES and HERP families: multiple effectors of the notch signaling pathway. J Cell Physiol 2003; 194 : 237-55.

[6] Bolos V, Grego-Bessa J, De La Pompa JL. Notch signaling in development and cancer. Endocr Rev 2007; 28: 339-63.

[7] Mitsiadis TA, Lardelli M, Lendahl U, Thesleff I. Expression of Notch 1, 2 and 3 is regulated by epithelial mesenchymal interactions and retinoic acid in the developing mouse tooth and associated with determination of ameloblast cell fate. J Cell Biol 1995; 130: 407-18.

[8] He F, Yang Z, Tan Y, et al. Effects of Notch ligand delta1 on the proliferation and differentiation of human dental pulp stem cells in vitro. Arch Oral Biol 2009; 54: 216-22.

[9] Mitsiadis TA, Romeas A, Lendahl U, Sharpe PT, Farges JC. Notch 2 protein distribution in human teeth under normal and pathological conditions. Exp Cell Res 2003; 282: 101-9.

[10] Lovscall H, Tummers M, Thesleff I, Fuchtbauer EM, Poulsen K. Activation of the Notch signaling pathway in response to pulp capping of rat molars. Eur J Oral Sci 2005; 113: 312-7.

[11] Kumamoto H, Ohki K. Detection of Notch signaling molecules in ameloblastomas. J Oral Pathol Med 2008; 37: 228-34.

[12] Casey LM, Lan Y, Cho ES, Maltby KM, Gridley T, Jiang R. Jag2Notch1 signaling regulates oral epithelium differentiation and palate development. Dev Dyn 2006; 235: 1830-44.

[13] Leethanakul C, Patel V, Gillespie J, et al. Distinct pattern of expression of differentiation and growth-related genes in squamous cell carcinomas of the head and neck revealed by the use of laser capture microdissection and cDNA arrays. Oncogene 2000; 19: $3220-4$. 
[14] Nakano K, Siar CH, Tsujigiwa H, Nagatsuka H, Nagai N, Kawakami T. Notch signaling in benign and malignant amelobastic neoplasms. Eur J Med Res 2008; 13: 476-80.

[15] Orstavik D, Mjor IA. Histopathology and X-ray microanalysis of the subcutaneous tissue response to endodontic sealers. J Endod 1988; 14: 13-23.

[16] Faratzis G, Tsiambas E, Rapidis AD, et al. VEGF and ki 67 expression in squamous cell carcinoma of the tongue: an immunohistochemical and computerized image analysis study. Oral Oncol 2009; 45: 584-8.

[17] Tsiambas E, Stamatelopoulos A, Karameris A, et al. Simultaneous EGFR and VEGF. alterations in non-small cell lung carcinoma based on tissue microarrays. Cancer Informat 2007; 3: 275-84.

[18] Jarriault S, Le Bail O, Hirsinger E, et al. Delta-1 activation of notch-1 signaling results in HES-1 transactivation. Mol Cell Biol 1998; 18: 7423-31.

[19] Takeuchi T, Adachi Y, Ohtsuki Y. Skeletrophin, a novel ubiquitin ligase to the intracellular region of Jagged-2, is aberrantly expressed in multiple myeloma. Am J Pathol 2005; 166: 1817-26.

[20] Mazella J, Liang S, Tseng L. Expression of delta-like protein 4 in the human endometrium. Endocrinology 2008; 149: 15-9.

[21] Sakamoto K, Ohara O, Takagi M, Takeda S, Katsube K. Intracellular cell-autonomous association of Notch and its ligands: a novel mechanism of Notch signal modification. Dev Biol 2002; 241: 31326.

[22] Shimizu K, Chiba S, Hosoua N, et al. Binding of Delta1, Jagged1, and Jagged2 to Notch2 rapidly induces cleavage, nuclear translocation, and hyperphosphorylation of Notch2. Mol Cell Biol 2000; 20 6913-22.

[23] Weinmaster G. The ins and outs of Notch signaling. Mol Cell Neurosci 1997; 9: 91-102.

[24] Osborne B, Miele L. Notch and the immune system. Immunity 1999; 11: 653-63.

[25] Zayzafoon M, Abdulkadir SA, Mcdonald JM. Notch signaling and ERK activation are important for the osteomimetic properties of prostate cancer bone metastatic cell lines. J Biol Chem 2004; 279: 3662-70.

[26] Tummers M, Thesleff I. Root or crown: a developmental choice orchestrated by the differential regulation of the epithelial stem cell niche in the tooth of two rodent species. Development 2003; 130: 1049-57.

[27] Chen WC, Tzeng YS, Li H. Gene expression in early and progression phases of autosomal dominant polycystic kidney disease. BMC Res Notes 2008, 1: 131.

Received: November 25, 2009

(C) Meliou et al.; Licensee Bentham Open.

This is an open access article licensed under the terms of the Creative Commons Attribution Non-Commercial License (http://creativecommons.org/licenses/by-nc/3.0/) which permits unrestricted, non-commercial use, distribution and reproduction in any medium, provided the work is properly cited. 